\title{
Preparation and Magnetic Properties of a Neutral Octadecanuclear Manganese Complex $\left[\mathrm{Mn}^{\mathrm{II}}{ }_{4} \mathrm{Mn}^{\mathrm{III}}{ }_{14}(\mathrm{O})_{14}\left(\mathrm{O}_{2} \mathrm{CMe}\right)_{18}(\mathrm{hmp})_{4}(\mathrm{hmpH})_{2}\left(\mathrm{H}_{2} \mathrm{O}\right)_{2}\right]$
}

\author{
Akhilesh K. Gupta, Seokwon Yoon, ${ }^{\dot{7}}$ Myoung Soo Lah,, and Jinkwon Kim \\ Department of Chemistru, Kongiu National Lniversity, 182 Shinkwan, Kongilt, Chungnam 314-701, Korea \\ "Department of Physics, The Catholic University of Korea, 43-1 Yoggok, Puchon 420-743, Korea

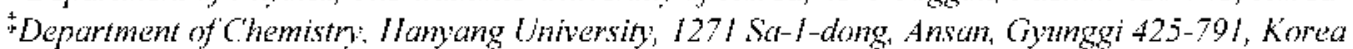 \\ Received.Januany 15,2004
}

Key Words : Manganese complex, Metal cluster, Magnetic properties

The synthesis of a magnetic molecule having unusually large spin value and large negative anisotropy value is an area of intensive current research, because it is the prerequisite for developing the emerging class of single-molecule magnets (SMMs). SMMs, nanometer-size single-domain magnetic clusters, have been found to display intramolecular magnetic hysteresis loop due to very slow magnetic relaxation below their blocking temperature as well as steps in the hysteresis loop assigned to the presence of quantum tunneling of the magnetization.' Since the dodecanulear manganese cluster with the composition $\left[\mathrm{Mn}_{12} \mathrm{O}_{12}\left(\mathrm{O}_{2} \mathrm{CMe}\right)_{16-}\right.$ $\left.\left(\mathrm{H}_{2} \mathrm{O}\right)_{4}\right]\left(\mathrm{Mn}{ }_{2} \mathrm{ac}\right)$ had been discovered as an singlemolecule magnet. ${ }^{2.3}$ many efforts have been inade to achieve larger cluster compounds showing SMM behaviors. ${ }^{4}$ Especially, manganese carboxylate cluster chemistry has proved to be a rich source of a variety of polynuclear species. ${ }^{5.6}$ Specific examples of SMMs except Mnl2ac include the tetranuclear cubane $\left[\mathrm{Mn}^{[1} \mathrm{Mn}^{1 / 1}{ }_{3} \mathrm{O}_{3} \mathrm{X}\right]^{6+}$ core $^{7}$ and $\left[\mathrm{Fe}_{4} \text { (sae) }\right)_{4-}$ $\left.(\mathrm{MeOH})_{4}\right]\left(\right.$ sae $=2$-salicylidene-amino- $\mathrm{I}$-ethanol) ${ }^{8}$ the octanuclear $\mathrm{Fe}(\mathrm{II})$ cluster $\left[\mathrm{Fe}_{8} \mathrm{O}_{2}(\mathrm{OH})_{12}(\operatorname{tacn})_{6}\right]^{8-}$ (tacn $=$ tetraazacyclononane) ${ }^{9}$ and the tetranuclear butterfly complex $\left[\mathrm{V}_{4} \mathrm{O}_{2}\left(\mathrm{O}_{2} \mathrm{CR}\right)_{7}(\mathrm{~L})_{2}\right] \mathrm{n}+\left(\mathrm{L}=\right.$ bipyridine or picolinate). ${ }^{10}$ Recently, a new family of manganese-based SMMs, such as [Mn?$\left.(\mathrm{OH})_{3} \mathrm{Cl}_{3}\left(\mathrm{~h}_{\mathrm{mp}}\right)_{6}\right]^{3+}$ and $\left[\mathrm{Mn}_{12} \mathrm{O}_{8} \mathrm{X}_{4}\left(\mathrm{O}_{2} \mathrm{Cl}\right)_{8} \mathrm{~L}_{8}\right]$, has been designed by using of hinp-bridging ligand (hınpH $=2$ hydroxymethylpyridine). ${ }^{11,12} \mathrm{On}$ the other hand, oxidation of $\mathrm{Mn}(\mathrm{II})$ by $\mathrm{MnO}_{4}^{-}$in $\mathrm{CH}_{2} \mathrm{Cl}_{2}$ leads to $\mathrm{Mn}_{2}{ }_{2} \mathrm{Mn}^{\mathrm{III}}$ chain complex instead of $\mathrm{Mnl} 2$ cluster. $^{1.3}$ Thus we have been trying new oxidation reaction of $\mathrm{Mn}(\mathrm{II})$ by $\mathrm{MnO}_{4}{ }^{-}$in a presence of hmpH and obtained a new octadecanuclear mixed-valent $\mathrm{Mn}$ cluster of formula $\left[\mathrm{Mn}_{18} \mathrm{O}_{14}\left(\mathrm{O}_{2} \mathrm{CMe}\right)_{18-}\right.$ (hmp) $\left.4(\mathrm{hmpH})_{2}\left(\mathrm{H}_{2} \mathrm{O}\right)_{2}\right](1)$.

The synthesis of 1 was achieved by reaction of an aqueous slurry of one equivalent of $\mathrm{Mn}\left(\mathrm{O}_{2} \mathrm{CCCH}_{3}\right)_{2} \cdot 4 \mathrm{H}_{2} \mathrm{O}$ in methylene chloride, 2.5 equivalents of hıpH, 0.5 equivalents of $\mathrm{NBu}_{4}$ " $\mathrm{MnO}_{4}$ in a presence of carboxylic acid. $\mathrm{NBu}_{4}$ "MnO $\mathrm{O}_{4}$ oxidizes $\mathrm{Mn}^{\text {"l }}$ to $\mathrm{Mn}^{\text {Ilt }}$ in presence of water and carboxylic acid.

An ORIEP diagram of 1 with atom labeling scheme is displayed in Figure 1. The centrosymmetric complex 1

"Conesponding Author. Phone: +82-41-850-8496; Fax: +82-4l850-8479; c-mail: jkim@ikongju.ac.kr

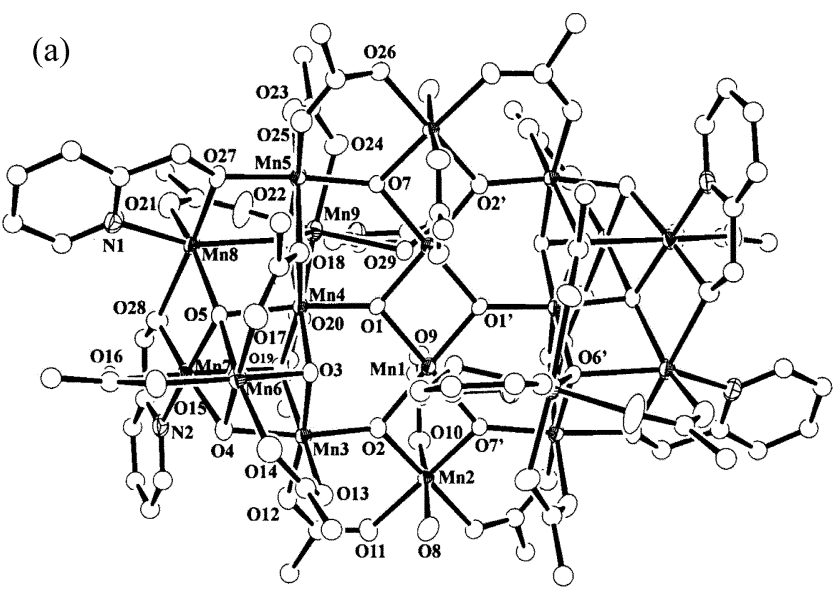

(b)

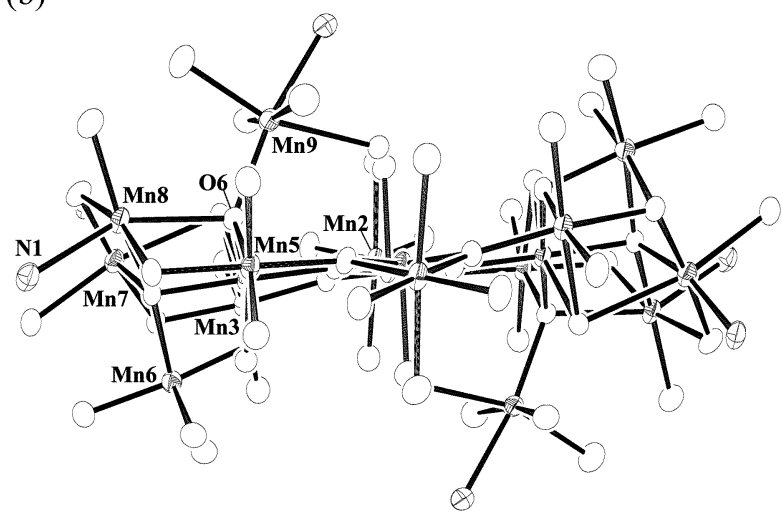

Figure 1. (a) ORILP diagram of 1 with atom numbering schems. (b) A side view emphasizing the planarity of $\left[\mathrm{Mnn}_{10} \mathrm{O}_{6}\right]$ unit.

consists of $\left[\mathrm{Mn}_{18}\left(\mu_{3}-\mathrm{O}\right)_{10}\left(\mu_{4}-\mathrm{O}\right)_{4}\right]$ core with peripheral chelation provided by eighteen acetate ligands and six $\mathrm{hmp}^{-}$ ligands, and two terminal water molecules. On the basis of Jahn-Teller distortions and bond valence sum calculations, the seven manganese atoms ( $\mathrm{Mn} 1-\mathrm{Mn} 7)$ in a crystallographically asymmetric unit were assigned to $\mathrm{Mn}^{\prime \prime \prime}$ and $\mathrm{Mn} 8$ and $\mathrm{Mn} 9$ to $\mathrm{Mn}{ }^{\prime \prime}$. The elongated axial $\mathrm{Mn}^{\prime \prime \prime}-\mathrm{O}$ distances (2.144(4)-2.456(4) $\AA$ ) are significantly longer than the other bonds $(1.854(3)-1.977(4) \AA)$. The $\mathrm{Mn}^{\mathrm{jl}}$-O bond distances are in a range from $2.104(4) \AA$ to $2.267(3) \AA$. As shown in the side view, ten manganese atoms from $\mathrm{Mn} 1$ to $\mathrm{Mn} 5$ and their 


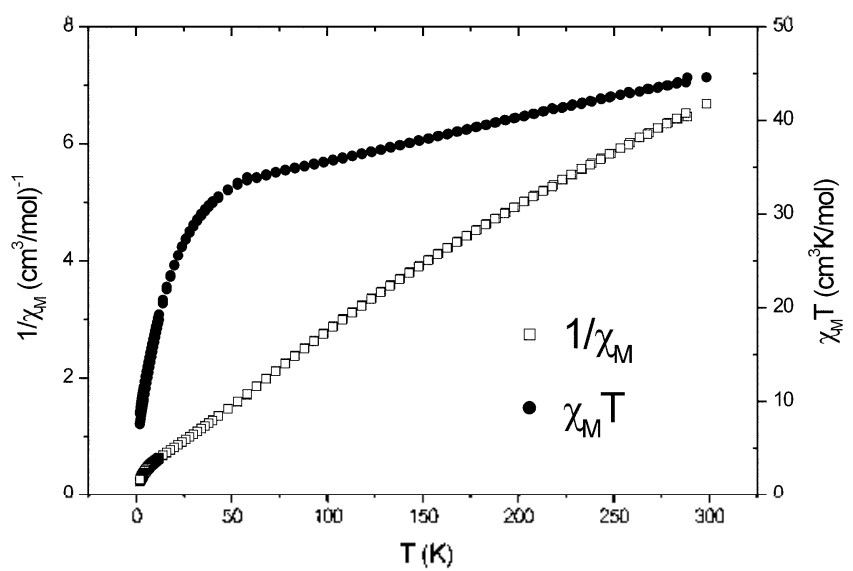

Figure 2. Temperature dependence of the $\chi_{N} T(\bullet)$ and $\left.1 / \chi_{u}(\lrcorner\right)$ for 1 at 10000 .

syınmetry related partners are almost co-planar and comprise a central planar $\left[\mathrm{Mn}_{10}\left(\mathrm{~m}_{3}-\mathrm{O}\right)_{6}\right]$ unit. This planar $\left[\mathrm{Mn}_{10}\left(\mathrm{I}_{3}-\mathrm{O}\right)_{6}\right]$ unit was also observed in the other $\mathrm{Mn}_{18}$ cluster $\left.\left[\mathrm{Mn}_{18} \mathrm{O}_{16}\left(\mathrm{O}_{2} \mathrm{CPh}\right)_{22} \text { (phthalate }\right)_{2}\left(\mathrm{H}_{2} \mathrm{O}\right)_{4}\right]^{4-}$. $^{+4}$ [wo $\mathrm{Mn}^{111}$ ions $(\mathrm{Mn} 6, \mathrm{Mn} 7)$ are located above and below the $\left[\mathrm{Mn}_{10}\left(\mathrm{~m}_{3}-\right.\right.$ $\mathrm{O})_{6}$ ] plane and bridged by oxygen atoms to form a distorted cubane $\left[\mathrm{Mn}_{1}\left(\mathrm{O}_{\text {oxidc }}\right)_{3}\left(\mathrm{O}_{\text {curlowyllute }}\right)\right]$ unit $(\mathrm{Mn3}, \mathrm{Mn} 4, \mathrm{Mn} 6$. $\mathrm{Mn} 7)$. The distance (3.610(I) $\AA$ ) between two $\mathrm{Mn}^{\prime \prime}$ ions is quite longer than the other $\mathrm{Mn}-\mathrm{Mn}$ distances $(2.810(\mathrm{l})$ $3.21 \mathrm{l}(1) \AA$ ). The octahedral geometry around $\mathrm{Mn}(8)$ is severely distorted such that the trans N1-Mn-O6 angle becomes $142.3(1)^{\circ}$. Charge considerations require protonation of two of oxygen donating ligands. Careful examination of structural parameters reveals that $\mathrm{O} 29$ is protonated and quite close to $\mathrm{O} 9(2.622 \AA)$ due to hydrogen bonds.

Magnetic susceptibility experiments were carried out on a powder sample $\mathbf{1}$ dried under air. TGA experiment indicates ca. three $\mathrm{CH}_{2} \mathrm{Cl}_{2}$ molecules exist even in a air-dried sample. The magnetic susceptibility data as a function of temperature, measured with an applied filed of I KG by using a SQUID magnetometer are displayed in Figure 2. $\chi_{i 1} T$ decreases almost linearly from $44.6 \mathrm{emuK} / \mathrm{mol}$ at $298 \mathrm{~K}$ to

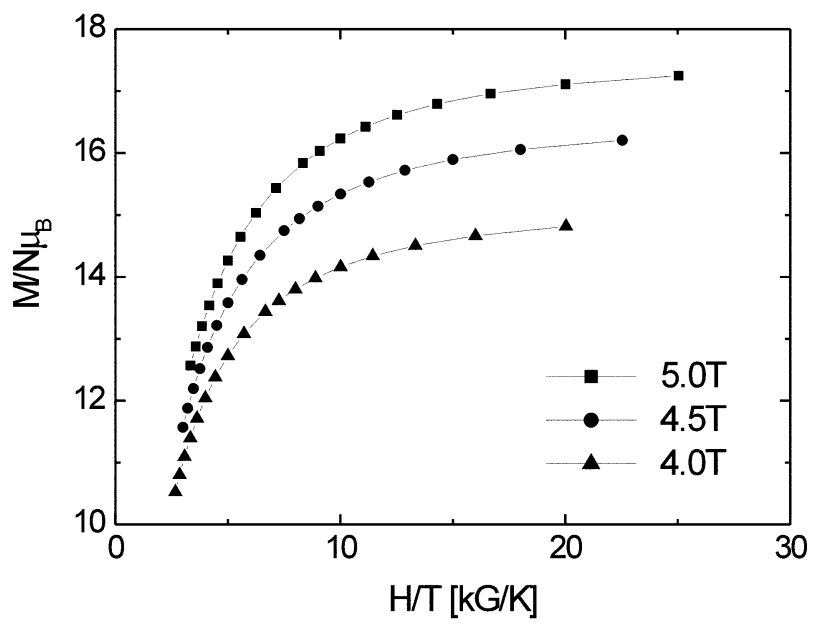

Figure 3. Plot of $W / N \mu_{\mathrm{B}} w / / T$ for 1 .
$33.6 \mathrm{emuK} / \mathrm{mol}$ at $58 \mathrm{~K}$. It is clear that there are strong intramolecular antiferromagnetic exchange interactions within complex 1 since $\chi_{N} T=59.5 \mathrm{emuK} / \mathrm{mol}$ is expected for an aggregate of noninteracting four $S=5 / 2$ and fourteen $S=2$. Below $\sim 50 \mathrm{~K}, \chi_{11} T$ drops faster down to $2.0 \mathrm{~K}(7.6$ emuK/ mol) indicating the existence of antiferromagnetic intermolecular interactions and/or zero-field splitting effects. ${ }^{15}$ Magnetization data were collected in the ranges $40-50 \mathrm{kG}$ and $2.0-10.0 \mathrm{~K}$ and the reduce magnetization $M / N_{H}$ is plotted as a function of $H / T$ in Figure 3 . The split of isofield lines shows that the zero-field splitting exists in the complex $\mathbf{l}$.

Also carried out were ac susceptibility measurements for the powder sample 1 in a $5.0 \mathrm{G}$ ac field oscillating at 250 $1000 \mathrm{~Hz}$ in the temperature range of $2.0-10 \mathrm{~K}$. Preliminary results showed no out-of-phase $\left(\chi_{i j}{ }^{\prime}\right)$ signals within the temperature range. However, G. Christou and his co-workers reported a new $\left[\mathrm{Mn}_{18}\right]^{2-}$ single-molecule magnet which has the same structure with our $\mathrm{Mn}_{18}$ complex but two $\mathrm{Mn}^{11}$ ions are further oxidized to $\mathrm{Mn}^{1 \mathrm{II}}$ compared to our $\mathrm{Mn}_{18}$ compound. ${ }^{16}$ The $\left[\mathrm{Mn}_{18}\right]^{2-}$ complex shows characteristics of single-molecule magnet such as temperature dependency of ac susceptibility in the $0.99-1.44 \mathrm{~K}$ region and magnetic hysteresis loop in the $0.04-1.0 \mathrm{~K}$. The total ground spin value was estimated as $\mathrm{S}=13$ which is unusually large spin for a molecular species. Furthermore, such SMM behaviors were observed in the smaller $\mathrm{Mn}_{12}$ cage which is also mixedvalent and consists of oxide and hınp ${ }^{-}$bridging ligand. ${ }^{12}$ Based on these recent results, we expect our $\mathrm{Mn}_{18}$ cluster may exhibit single-molecule magnetic behavior below $2.0 \mathrm{~K}$.

\section{Experimental Section}

General. Tetrabutylammonium permanganate was prepared by the previously reported methods. ${ }^{17.18}$ All other reagents were purchased from Aldrich Chemical Co. and Sigma Chemical $\mathrm{Co}$. and were used as received.

Elemental analyses $(\mathrm{C}, \mathrm{H}$ and $\mathrm{N}$ ) were performed by Fisons EA 1110 analyzer. IR spectra were recorded as compressed $\mathrm{KBr}$ discs on a Perkin Elmer Model 983 spectrophotometer in $4000-400 \mathrm{~cm}^{-1}$ range. Dc and ac Magnetic susceptibility measurements were carried out using a Quantum design MPMSYL susceptometer well equipped with a $5 \mathrm{~T}$ magnet and operating in the range 2.0 to $300 \mathrm{~K}$.

Synthesis. To an aqueous slurry of $\mathrm{Mn}$ (acetate $)_{2} \cdot 4 \mathrm{H}_{2} \mathrm{O}$ $(0.34 \mathrm{~g}, 1.63 \mathrm{mmol})$ in $1 \mathrm{~mL}$ water, a solution of hmpH $(0.4 \mathrm{~mL}, 4 \mathrm{mmol})$ in $20 \mathrm{~mL}$ of $\mathrm{CH}_{2} \mathrm{Cl}_{2}$ was added and followed by addition of $2 \mathrm{~mL}$ of acetic acid. The colorless reaction mixture was continuously stirred and treated with $\mathrm{NBu}_{4}{ }^{13} \mathrm{MnO}_{4}(0.29 \mathrm{~g}, 0.815 \mathrm{mmol})$ portionwise within an interval of $10 \mathrm{~min}$. The color of solution changes to dark brown. After $1 \mathrm{~h}$, the solvent was removed in vocuo and the oily residue was dissolved in $\mathrm{CH}_{2} \mathrm{Cl}_{2}$. Addition of equivolume of hexane and slow evaporation at room temperature for two weeks yielded crystals of 1 suitable for X-ray crystallography. Elemental analyses have been done with samples dried overnight in vacuo. Compound 1 : FTIR $(\mathrm{KBr}$, 
Table 1. Crystallographic data for complex 1

\begin{tabular}{|c|c|}
\hline Empirical formula & $\mathrm{C}_{78} \mathrm{H}_{108} \mathrm{Cl}_{12} \mathrm{M}_{118} \mathrm{~N}_{6} \mathrm{O}_{58}$ \\
\hline Molecular weight & 3472.02 \\
\hline Crystal system & Triclinic \\
\hline Space group & $P \bar{l}$ \\
\hline$a(\AA)$ & $14.411(1)$ \\
\hline$b(\AA)$ & $15.177(1)$ \\
\hline$c(\AA)$ & $15.729(1)$ \\
\hline$\alpha\left({ }^{\circ}\right)$ & $70.328(2)$ \\
\hline$\beta\left(0^{\circ}\right)$ & $78.407(2)$ \\
\hline$\gamma\left({ }^{\circ}\right)$ & $81.046(2)$ \\
\hline$F\left(\AA^{3}\right)$ & $3159.0(5)$ \\
\hline Z & 1 \\
\hline$P_{\text {calli }}\left(\operatorname{mg~} \mathrm{m}^{2}\right)$ & 1.825 \\
\hline$F(000)$ & 1736 \\
\hline$\mu\left(\mathrm{mm}^{-1}\right)$ & 2.072 \\
\hline Temperature $(\mathrm{K})$ & $173(2)$ \\
\hline $2 \theta_{\max }\left({ }^{\circ}\right)$ & 56.64 \\
\hline Transminssion factor & $0.4911-0.8196$ \\
\hline Reflections collected & 17933 \\
\hline Independent reflections & $13817\left[R_{\mathrm{gnt}}=0.0424\right]$ \\
\hline Observed reflections $[I>2 \sigma(I)]$ & 8549 \\
\hline No. of parameters & 815 \\
\hline$R_{1}, w R_{2}, \mathrm{GOF}$ & $0.0585,0.1349,1.006$ \\
\hline
\end{tabular}

Table 2. Selected Bond Distances $(\AA)$ and Angles ( $\left.{ }^{\circ}\right)$ for 1

\begin{tabular}{|c|c|c|c|c|c|}
\hline \multicolumn{6}{|c|}{ distances } \\
\hline Mnl & $-\quad O l$ & $1.948(3)$ & Mn1 & $-\mathrm{O} 2$ & $1.910(4)$ \\
\hline $\mathrm{Mnl}$ & - Ol & $1.920(3)$ & Mnl l & $-\mathrm{Ol}^{\prime}$ & $1.889(4)$ \\
\hline $\mathrm{Mnl}$ & - $\mathrm{O} 2$ & $1.919(4)$ & Mnl l & $-\quad O 7$ & $1.934(3)$ \\
\hline Mnl & -09 & $2.222(4)$ & $\mathrm{Mnl} 1$ & - $O 29^{\prime}$ & $2.455(4)$ \\
\hline $\mathrm{Mn} 2$ & - $\quad \mathrm{O} 2$ & $1.858(4)$ & $\mathrm{Mn} 2$ & $-\quad O 7$ & $1.865(4)$ \\
\hline $\mathrm{Mn} 2$ & - 08 & $2.328(4)$ & $\mathrm{Mn} 2$ & - Ol0 & $2.244(4)$ \\
\hline $\mathrm{Mn} 2$ & - Oll & $1.977(4)$ & $\mathrm{Mn} 12$ & $-026^{\circ}$ & $1.959(4)$ \\
\hline $\operatorname{Mn} \hat{3}$ & - $\quad 02$ & $1.886(4)$ & $\operatorname{Mn} 1 \hat{3}$ & -03 & $1.876(4)$ \\
\hline $\operatorname{Mn} \hat{3}$ & - $\mathrm{O}_{4}$ & $1.932(4)$ & $\operatorname{Mn} 1 \hat{3}$ & - $\mathrm{Ol} 2$ & $1.958(4)$ \\
\hline $\operatorname{Mn} 3$ & -013 & $2.187(4)$ & $\mathrm{Mn} 3$ & - 019 & $2.37 \mathrm{l}(4)$ \\
\hline Mnt & - $\mathrm{Ol}$ & $1.876(3)$ & Mn4 & -03 & $1.920(4)$ \\
\hline Mn4 & - 05 & $1.947(3)$ & Mn4 & -06 & $1.910(4)$ \\
\hline Mn4 & -018 & $2.237(4)$ & Mn14 & -019 & $2.291(4)$ \\
\hline Mnj & -06 & $1.903(3)$ & Mn15 & $-\quad 07$ & $1.891(3)$ \\
\hline Mns & - 018 & $2.422(4)$ & Mn15 & -023 & $2.144(4)$ \\
\hline $\operatorname{Mn} 5$ & -025 & $1.959(4)$ & Mn15 & - $\quad 027$ & $1.908(4)$ \\
\hline Mn6 & -03 & $1.880(4)$ & Mn6 & -04 & $1.942(4)$ \\
\hline Mn6 & -05 & $2.226(4)$ & Mn6 & - Ol4 & $2.180(4)$ \\
\hline Mno & -015 & $1.966(4)$ & Mn16 & - 017 & $1.964(4)$ \\
\hline $\mathrm{Mn} 7$ & -04 & $1.915(4)$ & $\mathrm{Mn} 7$ & $-O 5$ & $1.854(3)$ \\
\hline $\mathrm{Mn} 7$ & -016 & $2.174(4)$ & $\mathrm{Mn} 7$ & - 019 & $2.456(4)$ \\
\hline $\operatorname{Mn} 7$ & $\begin{array}{ll}- & 028\end{array}$ & $1.889(4)$ & $\operatorname{Mn} 7$ & $-\quad N 2$ & $2.043(5)$ \\
\hline $\operatorname{Mn} 8$ & - $\quad$ O5 & $2.197(4)$ & Mn8 & -06 & $2.267(3)$ \\
\hline $\operatorname{Mn} 8$ & $-\quad \mathrm{O} 2 \mathrm{l}$ & $2.104(4)$ & Mn18 & - $\quad 027$ & $2.136(4)$ \\
\hline $\operatorname{Mn} 8$ & - $\quad 028$ & $2.216(4)$ & Mn18 & - Nl & $2.244(5)$ \\
\hline $\operatorname{Mn} \varphi$ & -06 & $2.109(4)$ & Mn19 & -020 & $2.207(4)$ \\
\hline $\operatorname{Mn} 9$ & - $\quad 022$ & $2.128(4)$ & $\operatorname{Mn} 9$ & -024 & $2.190(4)$ \\
\hline $\operatorname{Mn} 9$ & - $\quad 029$ & $2.332(4)$ & $\operatorname{Mn} 9$ & $-N 3$ & $2.286(5)$ \\
\hline Mnl & ‥ Mnl' & $2.893(2)$ & Mnl 1 & $\cdots \mathrm{Mn2}$ & $2.810(1)$ \\
\hline $\mathrm{Mn} 3$ & ... Mnt & $3.082(1)$ & $\mathrm{Mn} 3$ & $\cdots \mathrm{Mn} 6$ & $2.818(1)$ \\
\hline $\operatorname{Mn} \hat{3}$ & $\begin{array}{ll}\cdots & M 17\end{array}$ & $3.210(1)$ & Mn14 & $\cdots M_{115}$ & $3.059(1)$ \\
\hline Mn4 & ... Mn16 & $2.940(1)$ & Mn14 & $\cdots \quad M_{11} 7$ & $3.21 \mathrm{l}(1)$ \\
\hline Mn4 & ... $\quad$ M118 & $3.119(1)$ & Mn15 & $\cdots \mathrm{Mn}_{18}$ & $3.203(1)$ \\
\hline Mn6 & $\cdots \quad \operatorname{Mn} 7$ & $2.840(1)$ & $\operatorname{Mn} 7$ & $\cdots \quad M n 8$ & $3.116(1)$ \\
\hline
\end{tabular}

Table 2. continuled

\begin{tabular}{|c|c|c|c|c|c|}
\hline \multicolumn{6}{|c|}{ angles } \\
\hline Ol & $11-\mathrm{Ol}^{\prime}$ & $81.15(15)$ & Ol & $\mathrm{Mnl}-\mathrm{O} 2$ & $99.36(15)$ \\
\hline $\mathrm{Ol}$ & O7' & $179.30(15)$ & $\mathrm{Ol}$ & $\mathrm{Mnl}-\mathrm{O} 9$ & $8907(14)$ \\
\hline $\mathrm{Ol}$ & $11-029^{\circ}$ & $90.75(13)$ & $\mathrm{Ol}$ & $\mathrm{Mnl}-07^{\prime}$ & $9908(15)$ \\
\hline $\mathrm{Ol}$ & [nl- 09 & $91.99(15)$ & Ol & - Mnl - O29' & $77.56(14)$ \\
\hline $\mathrm{O} 2$ & - Mnl - Ol' & $176.62(16)$ & $\mathrm{O} 2$ & $-\mathrm{Mnl}-O 7^{\prime}$ & $80.45(15)$ \\
\hline $\mathrm{O} 2$ & $-\mathrm{M}$ & $91.36(15)$ & $\mathrm{O} 2$ & - $\mathrm{MnI}-\mathrm{O} 29^{\prime}$ & $99.08(14)$ \\
\hline 07 & $11-09$ & $90.26(14)$ & 07 & - Mnl - O29' & $89.95(13)$ \\
\hline 09 & $11-029^{\circ}$ & $169.44(14)$ & $\mathrm{O} 2$ & $-\mathrm{Mn} 2-O 7^{\prime}$ & $83.89(15)$ \\
\hline $\mathrm{O} 2$ & -1 & $88.49(15)$ & $\mathrm{O} 2$ & $-\mathrm{Mn} 2-010$ & $94.77(15)$ \\
\hline $\mathrm{O} 2$ & $-\operatorname{Mr} 2$ & $94.72(16)$ & $\mathrm{O} 2$ & - $\mathrm{Mn} 2-026^{\prime}$ & $3(17)$ \\
\hline O7 & $-N$ & $95.18(16)$ & O7 & $-\mathrm{Mn} 2-010$ & $91.49(15)$ \\
\hline 07 & - Mn2-Oll & $178.60(16)$ & 07 & - Mn2- O26 & $96.94(16)$ \\
\hline 08 & $-\mathrm{Mn} 2-\mathrm{Ol} 0$ & $172.86(15)$ & 08 & - Mn2-Oll & $84.75(17)$ \\
\hline 08 & $026^{\circ}$ & $92.38(16)$ & 010 & - Mn2-Oll & $88.64(16)$ \\
\hline Olo & $-\operatorname{Mr2}$ & (16) & Oll & - $\mathrm{Mn} 2-\mathrm{O} 26^{\prime}$ & (16) \\
\hline $\mathrm{O} 2$ & $\mathrm{O} 3$ & $90.76(15)$ & $\mathrm{O} 2$ & $-\operatorname{Mn} 3-O 4$ & $168.99(17)$ \\
\hline $\mathrm{O} 2$ & - Mn3-O12 & $95.37(16)$ & $\mathrm{O} 2$ & - Mn3-Ol3 & $95.55(15)$ \\
\hline $\mathrm{O} 2$ & - Mn3-O19 & $87.74(15)$ & 03 & - Mn\}-O4 & $1(15)$ \\
\hline $\mathrm{O} 3$ & $\mathrm{O} 12$ & $173.33(15)$ & $\mathrm{O} 3$ & - Mn3-Ol3 & 15) \\
\hline $\mathrm{O} 3$ & - Mn13 - Ol9 & $2(14)$ & $\mathrm{O} 4$ & - $\operatorname{Mn} 3-O 12$ & 16) \\
\hline $\mathrm{O} 4$ & $13-\mathrm{Ol} 3$ & $93.52(16)$ & $\mathrm{O} 4$ & - Mn3-Ol9 & $8(14)$ \\
\hline 012 & - Mn3-O13 & $90.73(17)$ & $\mathrm{O} 12$ & - Mn3-O19 & $94,09(16)$ \\
\hline 013 & 019 & $173.89(14)$ & Ol & - Mn4- 03 & $9(15)$ \\
\hline Ol & - & $173.71(16)$ & $\mathrm{Ol}$ & - Mn4-06 & 15) \\
\hline Ol & - $\mathrm{Mn}$ & (14) & Ol & - Mn4-019 & 13) \\
\hline $\mathrm{O} 3$ & - Mr14- O5 & $86.31(15)$ & $\mathrm{O} 3$ & - Mn4- O6 & $174.45(15)$ \\
\hline 03 & - Mn4-018 & $88.14(14)$ & 03 & - Mn4-019 & $84.78(14)$ \\
\hline 05 & & $90.36(15)$ & 05 & - Mn4-018 & $8(14)$ \\
\hline 05 & 19 & $2(13)$ & 06 & - Mn4-O18 & 14) \\
\hline O6 & O19 & (14) & Ol8 & - Mn4 - O19 & 164 \\
\hline 06 & - Mn5-O7 & $91.49(15)$ & 06 & - Mn5-018 & $4(14)$ \\
\hline 06 & $n 5-023$ & 100. & 06 & - Mn5-O25 & $167.23(17)$ \\
\hline 06 & 227 & (15) & 07 & - Mn5- 018 & (14) \\
\hline O7 & - Mr & $(16)$ & O7 & - Mn5-O25 & 15) \\
\hline O7 & - Mri & $175.06(17)$ & $\mathrm{O} 18$ & - Mn5-O23 & (14) \\
\hline 018 & - Mn5-O25 & $86.93(15)$ & 018 & - Mn5-O27 & $87.14(15)$ \\
\hline $\mathrm{O} 23$ & - Mn5-O25 & $89.93(16)$ & $\mathrm{O} 23$ & - Mn5-O27 & $8(16)$ \\
\hline 025 & 27 & $(15)$ & 03 & - Mn6- & 15) \\
\hline $\mathrm{O} 3$ & - Mrr & (14) & 03 & - Mn6-Ol4 & 16) \\
\hline $\mathrm{O} 3$ & Mn16-OI5 & $174.99(16)$ & $\mathrm{O} 3$ & $-\operatorname{Mn} 6-017$ & (16) \\
\hline O4 & - Mn6- 05 & $80.14(14)$ & O4 & - Mn6-Olt & $93.14(16)$ \\
\hline O4 & $\ln 6-015$ & $94.23(16)$ & 04 & - Mn6-017 & $171.80(17)$ \\
\hline 05 & & (14) & O5 & 015 & \\
\hline 05 & - Mn16 & $2(15)$ & Ol4 & $-\operatorname{Mn} 6-015$ & 93 \\
\hline Ol4 & - Mn6-OI7 & $93.24(17)$ & Ols & $-\operatorname{Mn} 6-017$ & 90. \\
\hline O4 & - Mn7-O5 & $91.15(16)$ & O4 & $-\operatorname{Mn} 7-016$ & $89+41(16)$ \\
\hline O4 & $\mathrm{Mn} 7-\mathrm{O} 28$ & $174.52(17)$ & O4 & $-\operatorname{Mn} 7-N 2$ & $2(18)$ \\
\hline 05 & 16 & $15)$ & 04 & $7-019$ & 14) \\
\hline 05 & - Mn17-Ol9 & (14) & 05 & $-\operatorname{Mn} 7-O 28$ & \\
\hline 05 & $-\mathrm{Mn} 7-\mathrm{N} 2$ & $165.52(18)$ & Ol6 & - $\mathrm{Mn} 7-\mathrm{O} 28$ & $5(17)$ \\
\hline $\mathrm{Ol}$ & $-\mathrm{Mn} 7-019$ & $167.35(14)$ & $\mathrm{Olt}$ & $-\operatorname{Mn} 7-N 2$ & $97.28(17)$ \\
\hline Ol & $-\mathrm{Mn} 7-\mathrm{O} 28$ & $93.86(15)$ & 019 & $\mathrm{Mn} 7-\mathrm{N} 2$ & $7(15)$ \\
\hline $\mathrm{O}^{2}$ & & $18 \mathrm{j}$ & 05 & - Mn8-O6 & $7(13)$ \\
\hline O5 & $\mathrm{O} 21$ & 156. & O5 & - Mn8 - 027 & 97. \\
\hline $\mathrm{O} 5$ & $-\mathrm{Mn} 8-\mathrm{O} 28$ & (13) & 05 & - Mn8- Nl & 96 \\
\hline O6 & - Mn8-O21 & $103.73(15)$ & 06 & $-\operatorname{Mn} 8-027$ & (13) \\
\hline O6 & - Mn8-O28 & $115.22(14)$ & 06 & - Mn8 & $142.40(15)$ \\
\hline $\mathrm{O}^{2}$ & 027 & $104.27(16)$ & $\mathrm{O} 2$ & - Mn8- O28 & $87.07(16)$ \\
\hline $\mathrm{O} 2$ & $-\mathrm{M}$ & $97.47(17)$ & $\mathrm{O} 27$ & - Mn8-O28 & $165.72(15)$ \\
\hline $\mathrm{O} 2$ & - Mn8-Nl & $73.86(16)$ & $\mathrm{O} 28$ & - Mn8-Nl & $9636(16)$ \\
\hline O6 & - Mn9-O20 & $91.62(15)$ & 06 & - Mn9-O22 & $102.09(16)$ \\
\hline 06 & $\mathrm{Mn} 9-\mathrm{O} 24$ & $99.78(15)$ & 06 & $\mathrm{O} 29$ & $97.44(13)$ \\
\hline O6 & N3 & $167.47(16)$ & $\mathrm{O} 20$ & - Mn9-O22 & $90.68(19)$ \\
\hline $\mathrm{O} 2 \mathrm{C}$ & - Mr19-O24 & $168.49(15)$ & $\mathrm{O} 2 \mathrm{C}$ & - Mn9-O29 & $89.57(14)$ \\
\hline $\mathrm{O} 2$ & - Mn9- N3 & $8304(17)$ & $\mathrm{O} 2$ & Mn9- O24 & $88.54(19)$ \\
\hline $\mathrm{O} 2$ & - Mn9-O29 & $160.45(16)$ & $\mathrm{O} 22$ & $\mathrm{Mn} 9-\mathrm{N} 3$ & $89.35(17)$ \\
\hline $\mathrm{O}_{2}$ & N3 & $85.46(17)$ & $U_{2}$ & Mn9- O29 & $87.34(15)$ \\
\hline & & & & & \\
\hline
\end{tabular}

stmmetry codes: $-\mathrm{x}-1 .-\mathrm{y}+1,-\mathrm{z}$ 
$\mathrm{cm}^{-1}$ ): 1609 (vs). 1576 (vs), 1541 (vs), 1419 (vs) 1341 (w). 718 (m). 668 (s). 617 (s). 554 (m). Anal. Calcd for $\mathrm{C}_{72} \mathrm{H}_{6} \mathrm{Mn}_{18} \mathrm{~N}_{6} \mathrm{O}_{58}$ : C. 29.19; H. 3.27: N. 2.83\%. Found: C. 29.45 : H. $3.33 ;$ N. 3.01

Crystal Structure Determination. Reflection data were collected on an Bruker SMART CCD diffractometer using monochromated Mo $\operatorname{Ko} \alpha(\lambda=0.71073 \AA)$ radiation. The data were integrated and scaled using SAINT software package. ${ }^{19}$ Collected data were corrected for absorbance using $\mathrm{SADABS}^{14}$ based upon the Laue symmetry using equivalent reflections. Structure was solved by direct method and refined by leastsquares calculations with the SHELXL-PLUS 5.05 software package. ${ }^{\lambda^{i}}$ The non-hydrogen atonts were refuned anisotropically and the geometrically restrainted hydrogen atonis were treated using appropriate riding model. A sumunary of the crystallographic paranteters and data is given in Table 1.

Acknowlegement. This work was supported by the NRL program of the Ministry of Science and Teclnnology, Korea. One of the authors (S.Y.) acknowledges the support by KOSEF via Electron Spin Science Center at POSTECH.

\section{References}

1. Caneschi. A: Gatteschi, A:; Sangregorio, C: Sessoli, R.: Sorace. L.: Connia. A.: Novak. M. A.: Paulsen. C.: Werndorfer. W. $J$. Magn Magn. Mater 1999.200.182.

2. Lis. T. Acta Cnstallogr 1980. B36.2042.

3. Sessoli. R.: Gatteschi. D. Camneschi. A.: Nowak; M. A. Nature 1993, 365,141
4. Aromi. G.; Aubin. S. M. J.: Bolcar. M. A.: Christou. G.; Eppley: H. I.: Folting. K.: Hendrickson. D. N.: Huttman. J. C.: Squire. R. C.: Tsai. H.-L.: Wang. S.: Wemple. M. W. Polhhdron 1998. 17 . 3005.

5. Park, C.-D.: Jung, D.-Y. Bull. Korean Chent. Soc. 2001. 22.611

6. Koo, B.-K.: Lee. U. Bull. Koman. Chem. Soc. 2001, 22, 103.

7. Aubin. S. M. J.: Spagna, S.; Eppley. H. J.: Sager. R. E.; Christou, G.: Hendrickson. D. N. Chem. Conmm. 1998. 803.

8. Oshio. H.: Hoshino. N.: Ito. Y. J. An. Chem. Soc. 2000. 122. 12602

9. Bara. A.-L.: Debrunner. P.; Gatteschi. D;: Schulz, Ch. E; Sessoli. R. Europhys. Lett. 1996. 35, 133

10. Castro. S. L: Sun, Z. M.: Grant. C. M.; Bollinger. J. C.: Hendrichson. D. N.: Christou. G. J. Am Chem. Soc. 1998. 120 . 2365.

11. Bolcar. M. A.: Aubin. S. M. T.: Folting. K.: Hendrickson. D. N.: Christou, G. Chem. Commm. 1997. 1485.

12. Boskovic, C.: Brechin, E. K: Streib. W. E: Folting. K.: Hendrickson, D. N.: Christou, G. Chem Commun. 2001, 467.

13. Kiml. J.: Lim. T. M.: Do. Y. Eur J. Horg Chem 2003. 2563.

14. Squire. R. C.: Aubin. S. M. I.: Folting. K.: Streib. W. E.: Hendrichison. D. N.: Christou. G. Angew. Chem. Int. Ed. Engl. $1995.34,887$

15. Kahn, O. Holecular Hagnetism, VCH: New York, 1993; pp $14-$ 16

16. Brechinl. E. K: Boskovic. C.: Wernsdorfer. W.: Yoo. T.: Yamaguchi. A.: Sanudo. E. C.: Concolino. T. R.: Rheingold. A. L.: Ishimoto. H.: Hendrickson. D. N.: Christou. G. J. An. Chen. Soc. 2002, 124,9710

17. Aromi. G.: Bhaduri. S.: Artus, P.: Huffiman, J. C.: Hendrickson, D. N.; Christou, G. Polyhedron 2002, 21. 1779.

18. Vincent. J. B.: Chang. H. R.: Folting. K.: Hufiman. J. C.: Christou. G.: Hendricksont. D. N. J. Ant Chent. Soc. 1987. 109. 5703.

19. Briker. ALS: Madison. WI. 1997.

20. Blessing. R. H. Acta Chstallogr: Sect A 1995, 51, 33. 